\title{
Refining Prognosis for Intracerebral Hemorrhage by Early Reassessment
}

\author{
Matthew B. Maas Brandon A. Francis Rajbeer S. Sangha Bryan D. Lizza
}

Eric M. Liotta Andrew M. Naidech

Department of Neurology, Northwestern University, Chicago, IL, USA

\author{
Key Words \\ Prognosis - Critical care outcomes - Patient outcome \\ assessment $\cdot$ Intracerebral hemorrhage $\cdot$ Neurochecks
}

\begin{abstract}
Background: Prognostic assessments, which are crucial for decision-making in critical illnesses, have shown unsatisfactory reliability. We compared the accuracy of a widely used prognostic score against a model derived from clinical data obtained 5 days after admission for patients with intracerebral hemorrhage (ICH), a condition for which prognostication has proven notoriously challenging and prone to bias. Methods: Patients enrolled in a prospective observational cohort study of spontaneous ICH underwent hourly Glasgow Coma Scale (GCS) assessment. Outcome was measured at 3 months using the modified Rankin Scale (mRS). We analyzed the change in correlation between GCS and 3-month mRS scores from admission through day 5, and compared the performance of a parsimonious set of day 5 clinical variables against the ICH score. Results: Data was collected on 254 subjects. The ICH score and day 5 GCS score were both correlated with 3-month mRS score $(p<0.001)$, but the correlation was stronger with day 5 GCS score ( $p<0.05$ by Fisher z-transformation). Premorbid mRS score, intraventricular hemorrhage and day 5 GCS score were independent predictors of outcome (all $p<0.05$ in ordinal regression model). While ICH score correctly classified good (mRS 0-3) vs. poor (mRS 4-6) outcome in 73\% of cases, the day 5 model correctly classified $83 \%$ of cases. Conclusions: A simple reas-
\end{abstract}

sessment after 5 days of care significantly improves the accuracy of prognosticating outcome in patients with $\mathrm{ICH}$. These data confirm the feasibility and potential utility of early reassessments in refining prognosis for patients who survive early stabilization of a severe neurologic injury.

(c) 2017 S. Karger AG, Basel

\section{Introduction}

Evaluating a patient's prognosis for survival and favorable recovery is a fundamental skill in neurology. Prognostic models based on admission severity variables are important as risk adjustment techniques in clinical research and quality indices [1-3]. At the bedside, questions about prognosis arise as families and care teams seek to establish the appropriateness of interventions and clarify the goals of care. At the time those decisions are made, hours to days of additional information about the patient's response to stabilization efforts are often available. This additional information may influence expected outcomes, but models that incorporate reassessments and additional medical information to refine prognostic impressions are not available.

Intracerebral hemorrhage ( $\mathrm{ICH})$, the most morbid form of stroke, is emblematic of the importance and limitations of prognostication as currently practiced [4]. Prognostication after ICH has notoriously been confounded by limited accuracy and "self-fulfilling prophesies" by which initial impressions lead to care limitations in cases with severe initial

\section{KARGER}

(c) 2017 S. Karger AG, Basel

E-Mail karger@karger.com

www.karger.com/ced
Matthew B. Maas, MD

Department of Neurology

710 N Lake Shore Drive, 11th Floor

Chicago, IL 60611 (USA)

E-Mail mbmaas@ northwestern.edu 
symptoms, perhapsinappropriately [5-7]. While the capacity for recovery maybe underestimated in somecases, major complications are likewise difficult to anticipate. Early neurologic deterioration due to hematoma growth, delayed intraventricular hemorrhage(IVH), worsening edema, and other factors are common early in the disease course, and associated with worse long-term outcomes [8-10].

Similar to other severe neurologic conditions, widely used prognostic models for ICH strongly influence treatment recommendations, but are based exclusively on admission data and thus cannot account for important changes that happen during the hospital course $[1,2,11]$. With that background in mind, a recent study tested the effect of full intensive supportive treatment and avoiding new do-not-resuscitate (DNR) orders during the first 5 days after $\mathrm{ICH}$, and found a meaningful reduction in observed mortality compared to ICH score predictions [12].

The goal of this study was to determine whether incorporating information acquired during the early phase of a patient's care improves the accuracy of prognostication compared to assessments based only on data available at the time of admission. Through an analysis that uses ICH as a model disease, we test the hypothesis that a prognostic model using an early neurologic reassessment predicts outcome more accurately than a widely used prognostic score derived from admission data.

\section{Methods}

Patients presenting to Northwestern Memorial Hospital with spontaneous ICH between January 2010 and March 2016 were prospectively enrolled in an observational cohort study. All cases were diagnosed by a board-certified vascular neurologist or neurointensivist utilizing CT and/or MRI. Patients with $\mathrm{ICH}$ attributed to trauma, hemorrhagic conversion of ischemic stroke, structural lesions, or vascular malformations were excluded. Patient on anticoagulant medications were included. The inclusion and exclusion criteria were consistent with those used in prior studies of prognosis after ICH $[1,2]$. All patients were admitted to a neuro/ spine-intensive care unit (NSICU) with a standard order set in the electronic order entry system. The Glasgow Coma Scale (GCS) score was prospectively recorded at the time of initial evaluation by a trained neurologist and/or neurosurgeon.

Demographic information, medical history, medication history, standardized clinical instruments (GCS, pre-ICH modified Rankin Scale [mRS]), pretreatment blood pressure, laboratory data, imaging data, medical management variables, surgical interventions, and medical complications were prospectively recorded. A certified examiner independent of the primary clinical team recorded the mRS score at 14 days or discharge, whichever came first, and ascertained the mRS score prospectively at 28 days and 3 months with a validated questionnaire $[13,14]$. Hematoma volumes were measured on industry standard DICOM images from both referring hospitals and ours using Analyze software (Mayo Clinic, Rochester, MN, USA) with a semi-automated process, a technique with high reliability that has been used as an end point in other ICH studies [15]. Our NSICU monitoring protocol, as previously reported in detail, included serial neurologic examinations (neurochecks) using the complete GCS performed by a neuroscience nurse on admission to the NSICU and hourly thereafter throughout the entire stay in the intensive care unit, and at least one repeat non-contrast head CT to assess for hematoma growth [16].

\section{Identification of Potential Prognostic Predictors}

The goal of the data analysis was to identify a parsimonious set of variables to predict outcome based on information gathered through the first 5 days of care, with particular attention to differentiating good vs. poor outcomes. In goals of care discussions about prognosis, decision-making often revolves around the concept of acceptably good vs. poor/undesirable outcome. For the sake of this study, we dichotomized the mRS as scores from 0 to 3 being good outcome and from 4 to 6 as poor outcome, which is consistent with many studies of ICH [17]. We excluded patients who died due to withdrawal of life support from the analysis to avoid the biases that have been reported in numerous prior studies.

Hematoma volume and the degree of neurologic impairment measured by the GCS have consistently emerged as the strongest predictors of outcome in numerous models and studies of ICH outcomes. We first determined whether there was a stronger correlation between initial or final ICH volume, and initial or day 5 GCS score (precisely, GCS score measured at $120 \mathrm{~h}$ after admission or the closest time prior) with the 3 -month $\mathrm{mRS}$ score by calculating Spearman correlation coefficient $(\rho)$. We compared correlation coefficients for statistical difference using Fisher $\mathrm{z}$-transformation. To further assess the predictive value of GCS score and ICH volume, and to identify other candidate predictors, we entered candidate variables into a logistic regression model to identify predictors of poor outcome ( $\mathrm{mRS}$ score $\geq 4$ ), and utilized a backward conditional selection technique which stepwise eliminated variables based on change in the likelihood ratio statistic, a technique we have used in prior studies to model predictors of outcome after ICH [18]. Measures of GCS score, hematoma volume, and IVH status were based on the most current assessment at day 5 .

We used an ordinal regression model to confirm that the variables identified as independently associated with 3-month good outcomes were predictive across the full spectrum of outcomes, not just at the mRS score 3 vs. 4 thresholds, again consistent with our prior studies of ICH outcomes and as advocated by other stroke outcomes experts $[10,18,19]$. We used the test of parallel lines to confirm that our data met the proportional odds assumptions for a valid ordinal model. As there is no uniformly accepted metric to compare the fit of logistic models or the performance of a test at correctly categorizing patients, we sought to compare 2 common metrics of model fit, namely, Nagelkerke R-squared and the $-2 \log$ likelihood, with 3 widely used measures of test discrimination, namely, the positive predictive value, negative predictive value, and the diagnostic effectiveness (the proportion of correctly classified subjects) [20].

The study was approved by the Institutional Review Board (IRB). Written informed consent was obtained from the patient or their legally authorized representative. The IRB approved a waiver of consent for patients who died during initial hospitalization or were incapacitated and for whom a legal representative could not be located. 
Fig. 1. The correlation between GCS and mRS scores is shown graphically by plotting the Spearman correlation between the 1- and 3-month mRS scores and serial GCS measurements from the time of admission through day 5 .

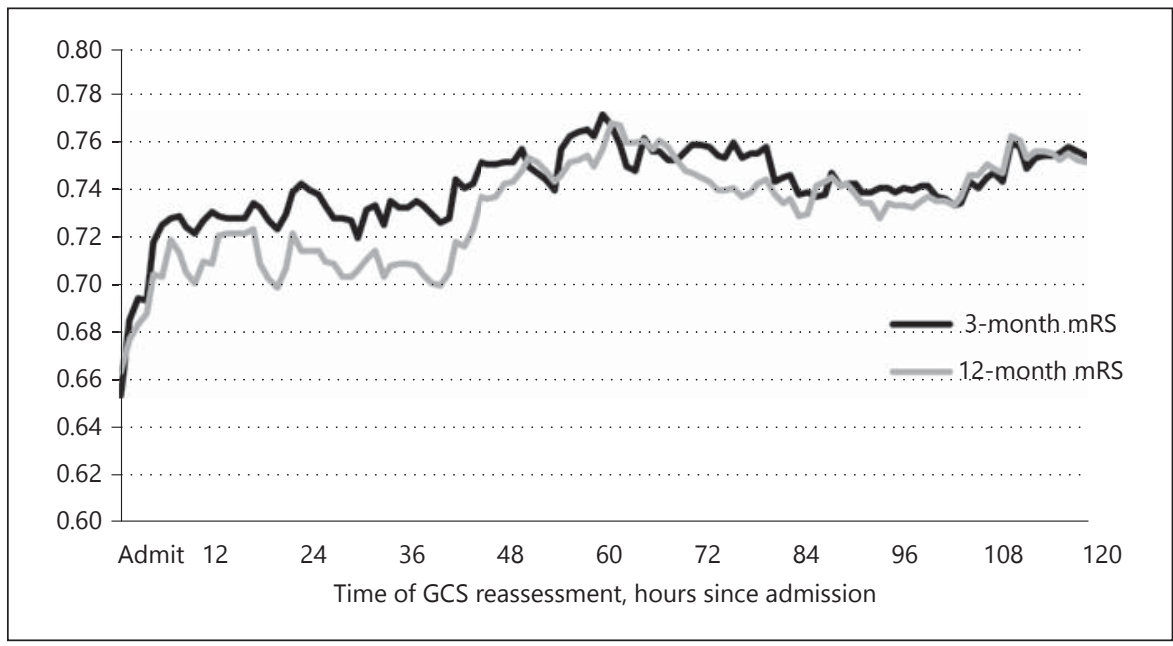

Table 1. Patient characteristics

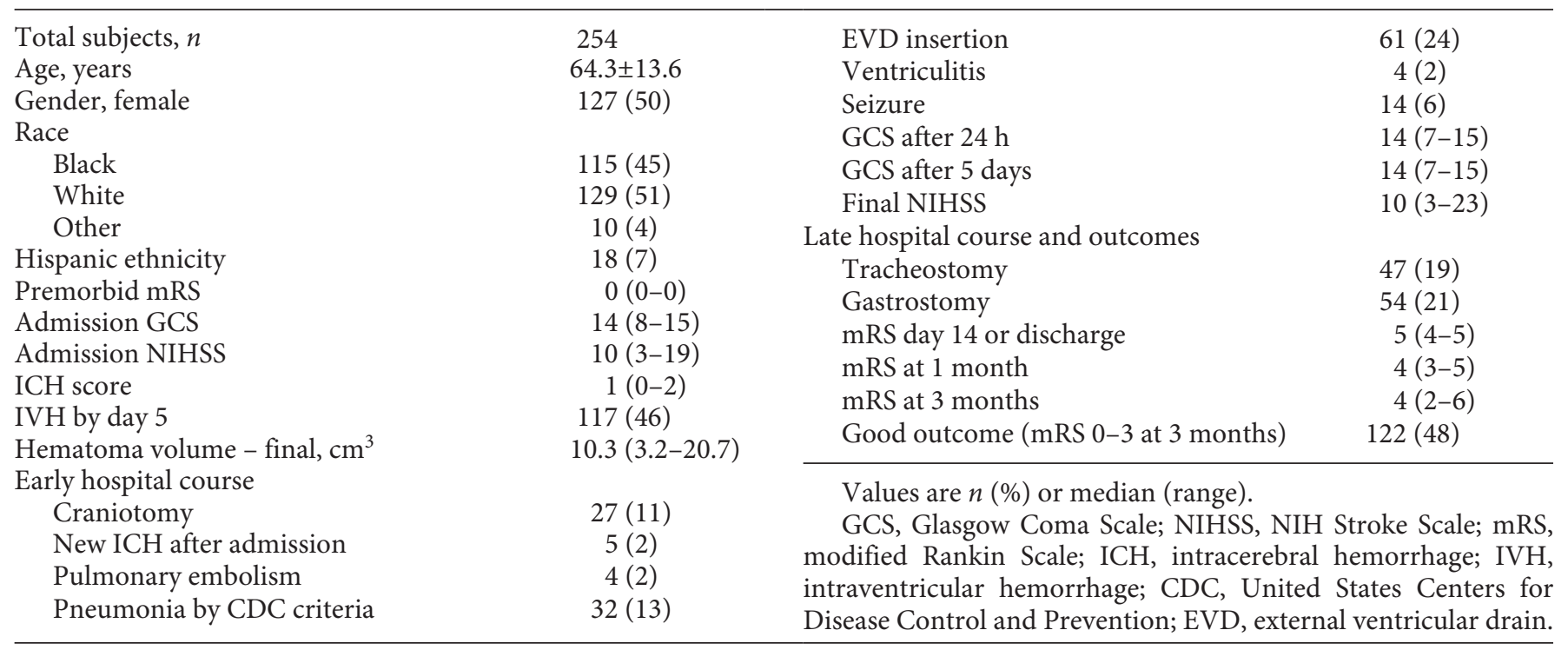

\section{Results}

We reviewed 460 patients with spontaneous $\mathrm{ICH}$ who met the clinical criteria. After excluding 69 for withdrawal of life support and another 135 in whom complete data were not available (mostly missing 3-month mRS score measurements), the study cohort comprised of 256 patients. The characteristics of those subjects, including major early hospital complications, are summarized in Table 1 . The only significant difference in measured baseline characteristics and early hospital course between included and excluded patients other than life support with- drawal was the final hematoma volume (median 10.3 vs. $14.2, p=0.02$; online suppl. Table 1, see www.karger.com/ doi/10.1159/000452679). GCS score correlated with 3 -month mRS score when measured at admission (Spear$\operatorname{man} \rho=-0.67, p<0.001$ ) and on day 5 (Spearman $\rho=$ $-0.76, p<0.001)$, but the correlation was stronger when GCS score was measured on day 5 ( $p=0.023$ for difference in Spearman correlation coefficient). The correlation between 3-month mRS score and day 5 GCS score was also stronger than between $\mathrm{mRS}$ and ICH scores (Spearman $\rho=-0.58, p=0.008$ ). The correlation between GCS and $\mathrm{mRS}$ scores is shown graphically in the Figure 1, which 
Table 2. Clinical factors independently associated with 3-month function outcome measured by the mRS

\begin{tabular}{lll}
\hline Variable & OR $(95 \% \mathrm{CI}), p$ value & \\
\cline { 2 - 3 } & $\begin{array}{l}\text { logistic model for dichotomized "poor outcome” } \\
\text { (mRS 4-6) }\end{array}$ & ordinal model for mRS \\
\hline Age & $1.03(0.99-1.06), p=0.102$ & $1.01(0.99-1.03), p=0.5$ \\
Premorbid mRS & $1.96(1.16-3.33), p=0.011$ & $1.68(1.18-2.38), p=0.004$ \\
IVH by day 5 & $2.38(1.03-5.56), p=0.041$ & $1.60(0.90-2.86), p=0.11$ \\
Hispanic ethnicity & $0.18(0.03-1.11), p=0.065$ & $0.28(0.10-0.80), p=0.018$ \\
GCS at day 5 & $0.63(0.54-0.72), p<0.001$ & $0.62(0.57-0.69), p<0.001$ \\
\hline
\end{tabular}

mRS, modified Rankin Scale; IVH, intraventricular hemorrhage; GCS, Glasgow Coma Scale.

plots the Spearman correlation between mRS and serial GCS measurements from the time of admission through day 5 . The relationship rapidly strengthens over $12 \mathrm{~h}$ and plateaus within the first 3 days. There was no significant difference between the correlations for 3-month mRS score and initial hematoma volume vs. final hematoma volume $(p=0.9)$.

Conditional variable selection by logistic modeling for 3-month good outcome led to the selection of age, Hispanic ethnicity, premorbid disability measured by $\mathrm{mRS}$, presence of IVH, and day 5 GCS score as potential predictors of outcome, with premorbid mRS, IVH, and GCS scores reaching statistical significance. The ordinal regression model confirmed a significant, independent association between 3-month $\mathrm{mRS}$ and premorbid $\mathrm{mRS}$ scores (OR 1.68, interquartile range 1.18-2.38, $p=0.004$ ), Hispanic ethnicity (OR 0.28 [0.10-0.80], $p=0.018$ ), and day 5 GCS score (OR 0.62 [0.57-0.69], $p<0.001$ ) across the range of outcomes measured by the mRS. The test of parallel lines did not reject the proportional odds assumption $(p=0.3)$, indicating a valid ordinal model. Results of the adjusted analyses are shown in Table 2. Post-hoc analysis found that Hispanic patients were significantly younger than non-Hispanics (mean age 58 vs. 65 years, $p=0.01$ ). Inclusion of an interaction term for Hispanic ethnicity and age did not change effect estimates for GCS or premorbid mRS scores, but Hispanic ethnicity became non-significant. No significant 2 -way interactions were identified.

Distilling these findings into figures that are tangible for clinicians making decisions at the bedside, the fully computed ICH score correctly categorized subjects in this cohort into good vs. poor outcome at 3 months in $73 \%$ of cases compared to $80 \%$ of cases correctly categorized by the day 5 GCS score alone or $83 \%$ with the full day 5 model. Both measures of model fit (Nagelkerke R-squared and
Table 3. Comparison of outcome prediction models by select model fit and test discriminatory indicators

\begin{tabular}{|c|c|c|c|}
\hline Metrics & $\begin{array}{l}\text { ICH } \\
\text { Score }\end{array}$ & $\begin{array}{l}\text { Day } 5 \\
\text { GCS }\end{array}$ & $\begin{array}{l}\text { Complete } \\
\text { day } 5 \\
\text { model }\end{array}$ \\
\hline \multicolumn{4}{|l|}{ Model fit } \\
\hline Nagelkerke R-squared & 0.36 & 0.52 & 0.46 \\
\hline$-2 \log$ likelihood & 270.7 & 170.0 & 148.1 \\
\hline \multicolumn{4}{|l|}{ Test discrimination } \\
\hline Positive predictive value, $\%$ & 66.7 & 75.5 & 79.1 \\
\hline Negative predictive value, $\%$ & 83.2 & 86.3 & 87.1 \\
\hline Diagnostic effectiveness & 0.73 & 0.80 & 0.83 \\
\hline
\end{tabular}

-2 log likelihood) and all 3 measures of test discrimination (positive predictive value, negative predictive value, and diagnostic effectiveness) showed that day 5 GCS score alone was superior to the ICH score in prognostication, and the complete day 5 model was superior to both, as summarized in Table 3. Similar magnitude differences are seen at other mRS cut points, for example, mRS scores of $0-4$ where ICH score correctly categorizes 80 vs. $89 \%$ for day 5 GCS score alone (NK-R ${ }^{2} 0.32$ and 0.50 , respectively).

\section{Discussion}

A simple re-evaluation is useful in refining the prognosis for patients with ICH. The correlation between GCS score and outcome strengthens over the first few days. By 5 days, GCS score alone outperforms the complete ICH score in correctly predicting good vs. poor outcome by a variety of definitions. Models based on information acquired after observing 5 days of treatment improve the 
prediction of good outcome by about $10 \%$ at various mRS thresholds compared to the ICH score, which comprises 5 variables measured at admission. These data provide an understanding of which factors best predict functional outcomes after ICH, reflecting not only the initial injury, but the response to stabilization and early therapies.

While models like the widely used ICH score will continue to play an important role as a risk adjuster for quality assessment, where the goal is to observe adjusted outcomes based on the patients' initial substrate, our findings suggest that a new perspective on prognostication as a process best refined in response to a patient's clinical trajectory may afford healthcare providers and families better guidance for care decisions as they happen along the course of hospitalization. Most neurologists and intensivists undoubtedly consider the patient's hospital course when estimating prognosis, although formal prognostic tools are important adjuncts to practitioner expertise, improving accuracy and diminishing bias [5]. A recent study that investigated prognosis and treatment recommendations by neurologists and neurosurgeons for $\mathrm{ICH}$ found that providing a prognostic estimate of the percent chance of functional independence significantly changed treatment recommendations, exerting a large influence on the likelihood of recommending treatment limitations [11]. In consequence, improving prognostic tools is imperative.

Recent research has found that changes in the first hours after hemorrhage onset affect outcomes, and can be accurately measured using standardized instruments such as the GCS that are already in widespread use as neurochecks $[16,21]$. This study extends those findings to show the effects of the early course of care. Three characteristics of the study data merit additional comment. First, the severity of neurologic deficits measured by the GCS score, already the most strongly correlated variable on admission, becomes an even better surrogate for outcome as time elapses. Perhaps this is because the other variables characterizing the brain injury that show independent effects at admission, like hematoma volume and hemorrhage location, are better reflected by the clinical examination as time goes on (i.e., collinear with GCS score). Second, the association between GCS score and functional outcome strengthens quickly over the first 12 $\mathrm{h}$, with minimal additional changes beyond 2.5 days. Although a previously mentioned study found better outcomes when DNR decisions were delayed for 5 days, these findings suggest that delaying half as long may allow for assessment of a patient's clinical trajectory that results in equally informed decision-making with respect to prog- nosis [12]. Finally, surgical interventions and infectious complications did not emerge as significant. It may be that this study is not sufficiently powered to identify their effects, or that surgical interventions and medical complications are much more likely to be observed in patients with high neurologic severity, such that GCS score remains a good surrogate for outcome.

Prognostic models must always be interpreted by expert clinicians when making decisions about individual cases, as uncommon factors that are nevertheless very important in individual cases are not well represented. For example, many models do not account well for comorbidities that limit life expectancy, although the presence of complicating factors like end stage metastatic cancer would obviously influence decision-making. To that end, there has been renewed interest in comparing the accuracy of experienced practitioners vs. empiric models. A recently published study found that practitioners are more accurate at predicting 3-month outcomes for patients with ICH than the ICH score, although practitioners in that study were allowed up to $24 \mathrm{~h}$ to reassess each case, raising the possibility that the opportunity to observe measurable, early clinical changes account for their findings, rather than practitioners' consideration of complex or immeasurable variables [22]. Moreover, the ability of expert practitioners to estimate prognosis, even with information about the early stabilization available, was poor. For example, $37.1 \%$ of patients in the previously mentioned study cohort died by 3 months, although practitioners had predicted death in only $14.5 \%$ of cases. Empiric prognostic models can provide a useful adjunctive to expert experience, guiding better decisions about appropriate care. The data we present here confirms the feasibility and utility of developing prognostic models based on the response to early treatment that offer a more accurate characterization of mortality risk and capacity for functional recovery.

Like prior studies modeling outcomes, this study has limitations. Our data are derived from a single center experience. Replicating our findings in another cohort would be useful. As noted earlier, clinical findings, comorbidities, and complications that occur at low frequency are less amenable to modeling, but may be very important in individual cases, which is an inherent limitation that will never allow quantitative models to substitute for physician judgment [23]. Moreover, the effect of some relevant baseline characteristics on outcomes (like premorbid functional status) may be more clearly seen over time, as shown in our day 5 model. Serum biomarkers relevant to prognosis are desirable, but none have entered 
routine clinical use [24, 25]. Ideally, prognostic models inform providers which common clinical factors influence outcomes with meaningful effect sizes. With a larger sized cohort, we may have been able to identify significant associations with complications that are less common or have smaller effect sizes. However, the cohort for this study is larger than the ones used to create the ICH score or to comparing the ICH score vs. practitioner estimates for prognosis $[22,26]$.

In conclusion, the prognostic value of commonly acquired clinical assessments like the GCS score increases in the days following hospital admission. This finding confirms that the response to resuscitation and early treatments is measurable and relevant to outcomes. As a result, simple prognostic models that use reassessed measures that reflect that response to resuscitation and stabilization provide a more accurate prediction of functional outcomes, shown in our study of ICH patients by incorporating a term for IVH that accounts for delayed IVH and a re-measure of the GCS score. Considering the momentous, life or death decisions that are made based on the likelihood of a good outcome, improving the accuracy with which good vs. poor outcome is predicted on the order of $10 \%$ is meaningful in practice. Perhaps more important are the potential applications for the principle underlying this proof of concept study. As electronic medical records and large data analytic techniques ad- vance, clinicians will increasingly rely on models that integrate real time clinical data to support their decisionmaking. This study supports the feasibility and utility of prognostic models that could refine estimates over several days for a variety of severe neurologic conditions, providing clinicians and families more reliable information to guide care.

\section{Funding}

Dr. Maas receives support from National Institutes of Health grants K23 NS092975 and L30 NS080176, and a Dixon Translational Research Grant from the Northwestern Memorial Foundation. Dr. Liotta receives support from the National Institutes of Health National Center for Advancing Translational Sciences grant KL2TR001424. Dr. Naidech receives support from Agency for Healthcare Research and Quality grant K18 HS023437. Research reported in this publication was supported, in part, by the National Institutes of Health's National Center for Advancing Translational Sciences grant UL1 TR000150. The content is solely the responsibility of the authors and does not necessarily represent the official views of the National Institutes of Health or the Agency for Healthcare Research and Quality.

\section{Disclosure Statement}

All authors declare that they have no conflicts of interest to disclose.

\section{References}

1 Hemphill JC 3rd, Farrant M, Neill TA Jr: Prospective validation of the ICH Score for 12-month functional outcome. Neurology 2009;73:1088-1094.

2 Rost NS, Smith EE, Chang Y, Snider RW, Chanderraj R, Schwab K, FitzMaurice E, Wendell L, Goldstein JN, Greenberg SM, Rosand J: Prediction of functional outcome in patients with primary intracerebral hemorrhage: the FUNC score. Stroke 2008;39:2304-2309.

3 Alonso A, Ebert AD, Kern R, Rapp S, Hennerici MG, Fatar M: Outcome predictors of acute stroke patients in need of intensive care treatment. Cerebrovasc Dis 2015;40:10-17.

4 Smith EE, Shobha N, Dai D, Olson DM, Reeves MJ, Saver JL, Hernandez AF, Peterson ED, Fonarow GC, Schwamm LH: A risk score for in-hospital death in patients admitted with ischemic or hemorrhagic stroke. J Am Heart Assoc 2013;2:e005207.

5 Becker KJ, Baxter AB, Cohen WA, Bybee HM, Tirschwell DL, Newell DW, Winn HR, Longstreth WT Jr: Withdrawal of support in intracerebral hemorrhage may lead to self-fulfilling prophecies. Neurology 2001;56:766-772.
6 Hemphill JC 3rd, Newman J, Zhao S, Johnston SC: Hospital usage of early do-not-resuscitate orders and outcome after intracerebral hemorrhage. Stroke 2004;35:1130-1134.

7 Zahuranec DB, Brown DL, Lisabeth LD, Gonzales NR, Longwell PJ, Smith MA, Garcia NM, Morgenstern LB: Early care limitations independently predict mortality after intracerebral hemorrhage. Neurology 2007;68:16511657.

8 Leira R, Dávalos A, Silva Y, Gil-Peralta A, Tejada J, Garcia M, Castillo J; Stroke Project, Cerebrovascular Diseases Group of the Spanish Neurological Society: Early neurologic deterioration in intracerebral hemorrhage: predictors and associated factors. Neurology 2004;63:461-467.

9 Dowlatshahi D, Demchuk AM, Flaherty ML, Ali M, Lyden PL, Smith EE; VISTA Collaboration: Defining hematoma expansion in intracerebral hemorrhage: relationship with patient outcomes. Neurology 2011;76:12381244.

10 Maas MB, Nemeth AJ, Rosenberg NF, Kosteva AR, Prabhakaran S, Naidech AM: Delayed intraventricular hemorrhage is common and worsens outcomes in intracerebral hemorrhage. Neurology 2013;80:1295-1299.

11 Zahuranec DB, Fagerlin A, Sanchez BN, Roney ME, Thompson BB, Fuhrel-Forbis A, Morgenstern LB: Variability in physician prognosis and recommendations after intracerebral hemorrhage. Neurology 2016;86: 1864-1871.

12 Morgenstern LB, Zahuranec DB, Sánchez BN, Becker KJ, Geraghty M, Hughes R, Norris G, Hemphill JC 3rd: Full medical support for intracerebral hemorrhage. Neurology 2015;84: 1739-1744.

13 Banks JL, Marotta CA: Outcomes validity and reliability of the modified Rankin scale: implications for stroke clinical trials: a literature review and synthesis. Stroke 2007;38:10911096.

14 Wilson JT, Hareendran A, Grant M, Baird T, Schulz UG, Muir KW, Bone I: Improving the assessment of outcomes in stroke: use of a structured interview to assign grades on the modified Rankin Scale. Stroke 2002;33:22432246. 
15 Naidech AM, Jovanovic B, Liebling S, Garg RK, Bassin SL, Bendok BR, Bernstein RA, Alberts MJ, Batjer HH: Reduced platelet activity is associated with early clot growth and worse 3 -month outcome after intracerebral hemorrhage. Stroke 2009;40:2398-2401.

16 Maas MB, Rosenberg NF, Kosteva AR, Bauer RM, Guth JC, Liotta EM, Prabhakaran S, Naidech AM: Surveillance neuroimaging and neurologic examinations affect care for intracerebral hemorrhage. Neurology 2013;81: 107-112.

17 Naidech AM, Beaumont JL, Berman M, Francis B, Liotta E, Maas MB, Prabhakaran S, Holl J, Cella D: Dichotomous "good outcome" indicates mobility more than cognitive or social quality of life. Crit Care Med 2015;43:16541659.

18 Maas MB, Nemeth AJ, Rosenberg NF, Kosteva AR, Guth JC, Liotta EM, Prabhakaran S, Naidech AM: Subarachnoid extension of pri- mary intracerebral hemorrhage is associated with poor outcomes. Stroke 2013;44:653-657.

19 Saver JL: Novel end point analytic techniques and interpreting shifts across the entire range of outcome scales in acute stroke trials. Stroke 2007;38:3055-3062.

20 Okeh UM, Okoro CN: Evaluating measures of indicators of diagnostic test performance: fundamental meanings and formulars. J Biomet Biostat 2012;3:132.

21 Maas MB, Berman MD, Guth JC, Liotta EM, Prabhakaran S, Naidech AM: Neurochecks as a biomarker of the temporal profile and clinical impact of neurologic changes after intracerebral hemorrhage. J Stroke Cerebrovasc Dis 2015;24:2026-2031.

22 Hwang DY, Dell CA, Sparks MJ, Watson TD, Langefeld CD, Comeau ME, Rosand J, Battey TW, Koch S, Perez ML, James ML, McFarlin J, Osborne JL, Woo D, Kittner SJ, Sheth KN: Clinician judgment vs formal scales for pre- dicting intracerebral hemorrhage outcomes. Neurology 2016;86:126-133.

23 Sato S, Delcourt C, Zhang S, Arima H, Heeley E, Zheng D, Al-Shahi Salman R, Stapf C, Tzourio C, Robinson T, Lindley RI, Chalmers J, Anderson CS; INTERACT2 Investigators: Determinants and prognostic significance of hematomasedimentationlevelsin acuteintracerebral hemorrhage. Cerebrovasc Dis 2016;41:80-86.

24 Senn R, Elkind MS, Montaner J, Christ-Crain M, Katan M: Potential role of blood biomarkers in the management of nontraumatic intracerebral hemorrhage. Cerebrovasc Dis 2014; 38:395-409.

25 Maas MB, Furie KL: Molecular biomarkers in stroke diagnosis and prognosis. Biomark Med 2009;3:363-383.

26 Hemphill JC 3rd, Bonovich DC, Besmertis L, Manley GT, Johnston SC: The ICH score: a simple, reliable grading scale for intracerebral hemorrhage. Stroke 2001;32:891-897. 